\title{
The Galactic thin and thick disks in the context of galaxy formation
}

\author{
Thomas Bensby ${ }^{1}$ and Sofia Feltzing ${ }^{2}$ \\ ${ }^{1}$ European Southern Observatory, Santiago, Chile; email: tbensby@eso.org \\ ${ }^{2}$ Lund Observatory, Lund, Sweden; email: sofia@astro.lu.se
}

\begin{abstract}
We have obtained high-resolution spectra and carried out a detailed elemental abundance analysis for a new sample of $899 \mathrm{~F}$ and $\mathrm{G}$ dwarf stars in the Solar neighbourhood. The results allow us to, in a multi-dimensional space consisting of stellar ages, detailed elemental abundances, and full kinematic information for the stars, study and trace their respective origins. Here we briefly address selection criteria and discuss how to define a thick disc star. The results are discussed in the context of galaxy formation.
\end{abstract}

Keywords. stars: abundances, stars: kinematics, Galaxy: abundances, Galaxy: disk

\section{Introduction}

The study of the Milky Way is important in the context of galaxy formation. The hierarchical build-up of galaxies within $\Lambda \mathrm{CDM}$, the currently most successful theory for the formation of large-scale structure in the Universe (e.g., Springel et al. 2006), implies that a galaxy like the Milky Way has suffered a bombardment of merging blocks over its life time. Stellar discs are very fragile to changes in the gravitational potential, hence the existence of spiral galaxies like the Milky Way is a challenge for $\Lambda$ CDM. However, recent studies by, e.g., Koda et al. (2009), Stewart et al. (2009), and Scannapieco et al. (2009), show that these effects might not be as severe as previously envisioned.

Evidence of the presence of a Galactic thick disc was first presented by Gilmore \& Reid (1983), showing that the density of stars as a function of distance from the Galactic plane towards the Galactic north pole could not be explained by a single exponential, but rather two exponentials with different scale heights and with different number densities in the plane. Subsequent observational studies have established that, (compared to the thin disc) the thick disc stellar population is a kinematically hotter population, it rotates more slowly, it is mainly an old stellar population (as old as the stellar halo), and that it has different chemical properties, particularly in the sense that it possesses higher $[\alpha / \mathrm{Fe}]$ ratios at a given metallicity (e.g., Bensby et al. 2003, 2005, 2007b; Reddy et al. 2006; Fuhrmann2008).

In the context of galaxy formation and as tests of models of galaxy formation, it is important to establish the properties of a disc system of the type observed in the Milky Way. Therefore, to fully understand why the Milky Way has two different disc populations, how they formed, how they evolved, and clarify their relationships to the stellar halo and the Galactic bulge we have carried out an extensive study of long-lived $\mathrm{F}$ and $\mathrm{G}$ dwarf stars in the Solar neighbourhood.

\section{A new stellar sample}

We have a sample of 899 nearby $\mathrm{F}$ and $\mathrm{G}$ dwarf stars for which we have obtained highresolution and high signal-to-noise spectra with several spectrographs (UVES, FEROS, 


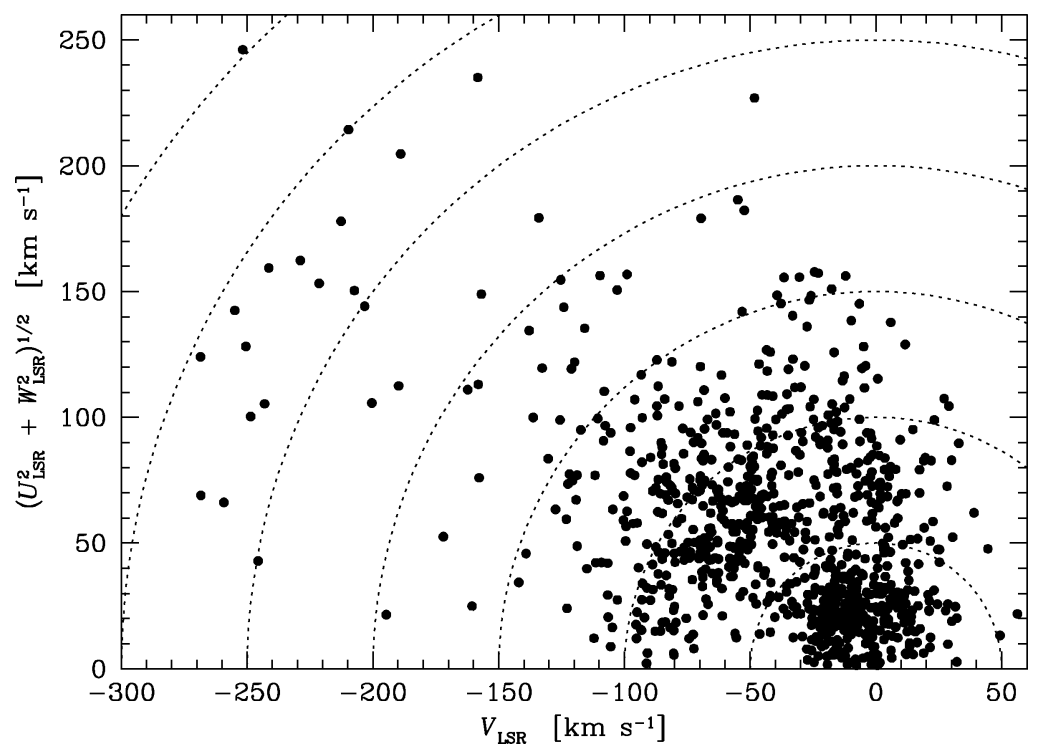

Figure 1. Toomre diagram. Dotted lines show constant values of the total space velocity, $v_{\text {tot }}=\sqrt{U_{\mathrm{LSR}}^{2}+V_{\mathrm{LSR}}^{2}+W_{\mathrm{LSR}}^{2}}$, in steps of $50 \mathrm{~km} \mathrm{~s}^{-1}$.

SOFIN, and MIKE; see Bensby et al. 2010, in prep. for details). Among the things we targeted during our observations are: (i) the oxygen trends in the metal-rich thin disc (Bensby et al. 2004); (ii) chemical differences between the thin and thick discs (Bensby et al. 2003, 2005); (iii) metal-rich and old stars; (iv) the metal-rich limit of the thick disc (first results in Bensby et al. 2007b); (v) the origin of the Hercules stream (first results in Bensby et al. 2007a); (vi) the metal-poor limit of the thin disc; (vii) the metal-poor limit of the thick disc; (viii) the stars with intermediate kinematics that can not be classified as neither thin disc nor thick disk stars (using current kinematical criteria, see Bensby et al. 2003, 2005); (ix) the metal-rich halo and its interface to the thick disc. Compared to our previous published thin and thick disc stellar sample that contained 102 dwarf stars (Bensby et al. 2003, 2005), the current sample is a factor $~ 8$ larger, and will allow us to put firmer observational constraints on the Galactic discs.

A Toomre diagram, which is a representation of the stars' combined vertical and radial kinetic energies as a function of the stars' rotational energy is shown in Fig. 1. Lowvelocity stars, constrained within $v_{\text {tot }} \equiv\left(U_{\mathrm{LSR}}^{2}+V_{\mathrm{LSR}}^{2}+W_{\mathrm{LSR}}^{2}\right)^{1 / 2} \lesssim 50 \mathrm{~km} \mathrm{~s}^{-1}$ are to a first approximation mainly thin disc stars, and stars with $v_{\text {tot }}$ greater than $\sim 70 \mathrm{~km} \mathrm{~s}^{-1}$, but less than $\sim 200 \mathrm{~km} \mathrm{~s}^{-1}$, are likely to be thick disc stars (e.g., Nissen 2004). Stars with higher velocities are halo stars. The Toomre diagram shows that our study sample the regions occupied by the thin and thick disc stellar populations well. There is also an excess of stars with velocities around $V_{\mathrm{LSR}} \sim-50 \mathrm{~km} \mathrm{~s}^{-1}$ and $\left(U^{2}+W^{2}\right)^{1 / 2} \sim 50-100 \mathrm{~km} \mathrm{~s}^{-1}$, the velocity space occupied by the Hercules stream (e.g., Bensby et al. 2007a).

\section{The dichotomy of the Galactic disc}

One of the aims with our study is to characterize the thin and thick discs in terms of detailed elemental abundances and stellar ages, and from there draw further conclusions about the origin and evolution of the two discs. As high-resolution observations of a large sample of $\mathrm{F}$ and $\mathrm{G}$ dwarf stars currently limits us to the stars of the immediate Solar 

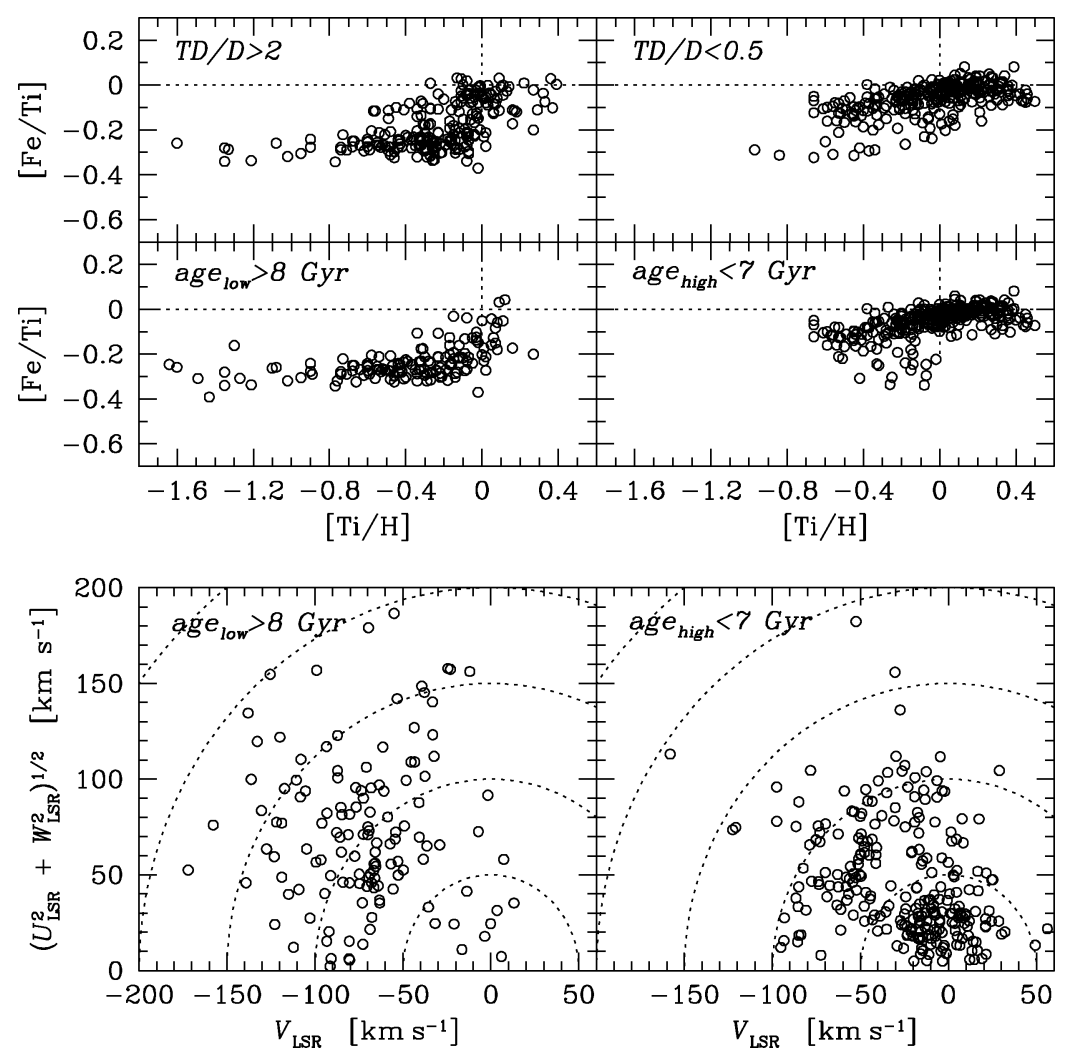

Figure 2. Thin disc (right-hand side) and thick disc (left-hand side) abundance trends using two different selection criteria. The stars in the top panels have been selected using the kinematical criteria used in Bensby et al. $(2003,2005)$, i.e., that a star has to be at least two times likely to belong to a stellar population than to any of the other populations. In the middle two panels the stars have been divided according to their ages: one sample with lower age estimates greater than $8 \mathrm{Gyr}$, and one sample with upper age estimates less than 7 Gyr. The two bottom panels show the Toomre diagrams for the old and young samples in the middle panels.

neighbourhood, typically the sphere covered by the Hipparcos mission, we have to rely on kinematical criteria when selecting our stellar sample. The top panels of Fig. 2, show the $[\mathrm{Fe} / \mathrm{Ti}]-[\mathrm{Ti} / \mathrm{H}]$ abundance trends for stars that are at least two times more likely of being thick disc stars than thin disc stars (top left panel), and vice versa in the top right panel. It is clear that these two sub-samples, only being different in terms of their kinematic properties, show quite different abundance trends. It is at the same time also clear that in each of these plots, there exist a number of stars that deviate from the large majority, and that actually would fit better with the other kinematic sample. This is most likely an effect of the fact that the velocity ellipsoids of the thin a thick discs partially overlap. Hence, when using kinematical criteria to pick either thin or thick disc stars we a likely to pick a few high-velocity thin disc stars in the thick disc sample, and a few low-velocity thick disc stars in the thin disc sample.

As most investigations indicate that the thick disc mainly is an old stellar population and that the thin disc is considerably younger, it might be illustrative to try and separate the sample by age criteria instead. This is what we have done in the middle panels of Fig. 2: one sample of stars whose ages have a lower age estimate of $8 \mathrm{Gyr}$ (left panel) and one sample of stars whose ages have an upper age estimate lower than 7 Gyr (right panel). 
Now we see two abundance trends very alike the ones we obtained by using kinematical criteria only. However, the number of "outliers" have been reduced and the abundance trends are "cleaner". The bottom panels in Fig. 2 show Toomre diagrams for these two age-selected samples and we see that the old sample mainly is a kinematically hot sample. The young sample, on the other hand, contains a lot of kinematically hot stars as well, stars that, when using kinematical criteria, would be selected as thick disc stars. This "kinematic confusion" demonstrates that one has to be careful when using kinematical criteria to select thin and thick disc stars, and that better criteria to separate the two populations are needed. However, Fig. 2 shows that, and if focus is not put on individual stars, kinematic criteria still can be used to obtain stellar samples of the Galactic stellar populations in order to trace their evolution.

\section{Discussion}

The mostly regarded scenario for the formation of the Galactic thick disc is that it is a result of an ancient merger event between the Milky Way and another (dwarf) galaxy (see, e.g., Quinn et al. 1993; Villalobos \& Helmi 2009). However, recent simulations by Schönrich \& Binney (2009) that show that it is possible to form a thick disc in the Galaxy, without a merger event, through radial mixing of gas and stars. Their model is able to reproduce all of the current observables (abundances, stellar ages, dichotomy of the Galactic disc, etc). So, currently, it appears as if the observable that would serve in favour of any these two formation scenarios is if there is a hiatus in the star formation history between the two discs. If present, the merger scenario would be favoured, as the model by Schönrich \& Binney (2009) implies a continuous star formation history.

\section{Acknowledgements}

Sofia Feltzing is a Royal Swedish Academy Research Fellow supported by a grant from the Knut and Alice Wallenberg Foundation.

\section{References}

Bensby, T., Feltzing, S., \& Lundström, I. 2003, A\& A, 410, 527

Bensby, T., Feltzing, S., Lundström, I., \& Ilyin, I. 2005, A\&A A, 433, 185

Bensby, T., Oey, M. S., Feltzing, S., \& Gustafsson, B. 2007a, ApJ, 655, L89

Bensby, T., Zenn, A. R., Oey, M. S., \& Feltzing, S. 2007b, ApJ, 663, L13

Fuhrmann, K. 2008, MNRAS, 384, 173

Gilmore, G. \& Reid, N. 1983, MNRAS, 202, 1025

Koda, J., Milosavljević, M., \& Shapiro, P. R. 2009, ApJ, 696, 254

Nissen, P. E. 2004, in Origin and Evolution of the Elements, Carnegie Observatories Astrophysics

Series, Vol. 4, (Eds.) A. McWilliam and M. Rauch, Pasadena: Carnegie Observatories, 156

Quinn, P. J., Hernquist, L., \& Fullagar, D. P. 1993, ApJ, 403, 74

Read, J. I., Lake, G., Agertz, O., \& Debattista, V. P. 2008, MNRAS, 389, 1041

Reddy, B. E., Lambert, D. L., \& Allende Prieto, C. 2006, MNRAS, 367, 1329

Scannapieco, C., White, S. D. M., Springel, V., \& Tissera, P. B. 2009, MNRAS, 396, 696

Schönrich, R. \& Binney, J. 2009, arXiv:090\%.1899v1 [astro-ph.GA]

Springel, V., Frenk, C. S., \& White, S. D. M. 2006, Nature, 440, 1137

Stewart, K. R., Bullock, J. S., Wechsler, R. H., \& Maller, A. H. 2009, ApJ, 702, 307

Villalobos, Á. \& Helmi, A. 2009, arXiv:0902.1624v1 [astro-ph.GA] 